\title{
The Precede-Proceed Model As Proposed Model In The One Health Paradigm
}

\author{
Oedojo Soedirham ${ }^{1} *$ \\ ${ }^{1}$ Faculty of Public Health, Universitas Airlangga \\ *oedojo-s@fkm.unair.ac.id or oedojo@yahoo.com
}

\begin{abstract}
One Health is "the collaborative effort of multiple disciplines - working locally, nationally, and globally — to attain optimal health for people, animals and the environment". One Health is a new phrase, but the concept extends back to ancient times. The recognition that environmental factors can impact human health can be traced as far back as to the Greek physician Hippocrates (c. 460 BCE - c. 370 BCE) in his text "On Airs, Waters, and Places". He promoted the concept that public health depended on a clean environment. "One Health" was mentioned in a story about Ebola hemorrhagic fever on April 7, 2003, when Rick Weiss of the Washington Post quoted William Karesh as saying, "Human or livestock or wildlife health can't be discussed in isolation anymore. There is just one health. And the solutions require everyone working together on all the different levels." "One Health" is an important global activity based on the concept that human, animal and environmental/ecosystem health are interdependent, and professionals working in these areas best serve the population by collaborating to better understand all the factors involved in disease transmission, ecosystem health, the emergence of novel pathogens and emerging zoonotic agents, as well as environmental contaminants and toxins that are capable of causing substantial morbidity and mortality, and impacting on socioeconomic growth, including in less developed countries. In another definition, the One Health Initiative Task Force (OHITF) defines one health as "the promotion, improvement, and defense for the health and well-being of all species by enhancing cooperation and collaboration between physicians, veterinarians, and other scientific health professionals and by promoting strengths in leadership and management to achieve these goals." By reviewing several documents including scientific papers, government documents, and other written resources this paper proposes the PRECEDE-PROCEED MODEL, a model to resolve the problems posed by the interaction between humans and animals.
\end{abstract}

Keywords: the one health paradigm, animal-human health collaborative links, the Precede-Proceed model.

\section{INTRODUCTION}

One health is a concept that aims to bring together human, animal, and environmental health. Researchers including Louis Pasteur and Robert Koch and physicians such as William Osler and Rudolph Virchow demonstrated the collaborative links between animal and human health. More recently, Calvin Schwabe revived the concept of One Medicine. As the traditional boundaries between medical and veterinary practice continue to pervade society there is a need for the practical application of one health ( $B$ i d a is e e a n d M a c p h e r o n, 2014). Nevertheless, although One Health as "the collaborative effort of multiple disciplines — working locally, nationally, and globally — to attain optimal health for people, animals and the environment" is a new phrase, but the concept extends back to ancient times. The recognition that environmental factors can impact human health can be traced as far back as to the Greek physician Hippocrates (c. 460 BCE - c. 370 BCE) in his text "On Airs, Waters, and Places". He promoted the concept that public health depended on a clean environment. 
This paper proposes a model to help solve health problems that arise because of the relationship between humans, animals and the environment that has been much discussed and researched.

\section{METHODS AND RESULTS}

By reviewing selected written papers and documents showing that the problem One Health is a complex problem and experienced by all countries (Bidaisee and Macpherson, 2014; Cutler, Fooks, and van der Poel, 2010; Galaz, Leach, Scoones, and Stein, 2015; National Institute Of Communicable Diseases, Delhi, 2005; Pan American Health Organization, 2003; Schneider, Aguilera, Smith, Moynihan, Barbosa da Silva, Aldighieri, 2011; van der Giessen, Isken, Tiemersma, 2004; World Health Organization, 2012).

Recenly, Schneider, Aguilera, Smith, Moynihan, Barbosa da Silva, Aldighieri (2011) and Macpherson, Meslin, Wandeler (2013) wrote that to date, $61 \%$ of human pathogens worldwide have been classified as zoonoses, a subgroup that comprises $75 \%$ of all emerging pathogens of the past decade. There is a growing belief - echoed in the "One World, One Health" strategic framework endorsed by the Food and Agriculture Organization (FAO), the World Organization for Animal Health (OIE), WHO, the United Nations Children's Fund (UNICEF), the United Nations System Influenza Coordination (UNSIC), and the World Bank - that an integrated approach to public health events is most effective and efficient with collaboration between physicians, veterinarians, and other health-related disciplines. Bidaisee and Macpherson (2014) reinforce the fact by quoting a statement that the one health approach plays a significant role in the prevention and control of zoonoses. It has been noted by the World Health Organization (WHO) (2005) and Graham et al. (2008) that approximately $75 \%$ of new emerging human infectious diseases are defined as zoonotic, meaning that they may be naturally transmitted from vertebrate animals to humans. New and reemerging zoonoses have evolved throughout the last three decades partly as a consequence of the increasing interdependence of humans on animals and their products and our close association with companion animals. Zoonoses should therefore be considered the single most critical risk factor to human health and well-being, with regard to infectious diseases.

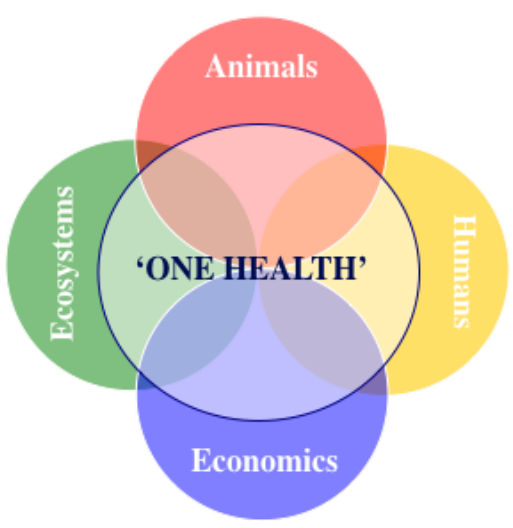

Figure 1. One Health as interlink among Humans, Animals, Ecosystems and Economics

Cripps (2000) in his personal perspectives stated that there are four main reasons why zoonoses need to be considered as serious threats to public health rather than merely as interesting examples of the natural history of some infectious agents. Firstly, a disease that starts as a zoonosis may have the potential to develop into a major human communicable disease. The use of elementary mathematical modelling suggests that for some communicable diseases the infection cannot sustain itself in the human populations below a critical minimum population size and density and that appropriate conditions were not met before the last few thousand years. As a result these communicable diseases must originally have been contracted from another species and hence 
be zoonoses. The conclusion from this is that zoonoses must be considered seriously as possible future human communicable diseases, and that ignoring them will pose a threat to public health. Secondly, many zoonoses are able to cause very significant human morbidity and mortality. Amongst these are brucellosis, leptospirosis, salmonellosis, tuberculosis and echinococcosis, and a large number of other bacterial, viral and parasitic infections. In many countries the impact of zoonotic disease has hardly been investigated at all so it is difficult to estimate their contribution to human illness. Thirdly, some zoonotic infections also cause serious disease in agricultural and food-producing animals. This can result in a reduced availability of animal-derived food and reduced wealth in animal-owning communities: and this in turn can adversely affect the level of public health. An example of this is tuberculosis. Many countries have made attempts to rid their animal populations of one or more zoonotic infections and in some cases these attempts have appeared to be successful. Experience has shown, however, that infections which have been reduced to a very low prevalence may be able, once control measures are relaxed, to reappear. This demonstrates the fourth reason that zoonoses are important: ignoring zoonoses does not cause them to go away, and new ones may emerge at any time. The only way to have any level of protection against them is to be constantly vigilant.

Furthermore, Cripps (2000) arguing that there are problems in research and education. His experience suggest that a lack of awareness amongst agencies which fund veterinary research, professionals in the scientific and health care communities and at ministerial level about the public health consequences of zoonotic disease. This lack of awareness may be partly due to deficiencies in education. Our students are used to the concept of transmission of diseases between species, and they know about infections that can be acquired by humans from animals. In addition they are taught how to alter the management and husbandry of animals in order to control disease. Veterinary graduates should therefore be ideally suited to deal with zoonoses. However even if they have the theoretical knowledge of the subject many of our veterinary graduates may not appreciate the relevance or importance of zoonoses. There can be little doubt that this situation is at least partly caused by the way that the subjects are taught. For example, teaching in Public Health maybe tends to refer almost exclusively to meat inspection, meat hygiene and food hygiene: little emphasis is placed on the veterinary role in wider aspects of public health such as zoonoses, water quality and human nutrition. Similarly, human health care professionals are often unused to including zoonoses in their list of differential diagnoses. In these circumstances it is hardly surprising that there tends to be a large degree of public ignorance of the risks of zoonotic infections and the ways to control and prevent them.

Cutler, Fooks, and van der Poel (2010) concluded that many zoonoses can be considered opportunistic infections. Increasing demands for protein necessitate increased levels of farming. Food can provide a vehicle for spread of pathogens from animals to humans. Contact with animals during farming, hunting, or by animal bites can increase transmission of diseases (e.g., rabies and tularemia). Arthropod vectors can transmit diseases on an immense scale to other hosts as in cases of West Nile fever and plague. Changing patterns of farming, life style, and transportation influence the dynamics of pathogen ecology. Pathogens are subjected to changes by many intrinsic and extrinsic factors. Mutation, recombination, selection, and deliberate manipulation can result in new traits acquired by pathogens and result in potential epidemic consequences. Reemergence of diseases through opportunistic host switching is likely to continue as a major source of human infectious disease. Strategies to improve public health have focused on improved surveillance in regions of perceived high likelihood of disease (reemergence). These strategies include improved detection of pathogens in reservoirs, early outbreak detection, broad-based research to identify factors that favor reemergence, and effective control (i.e., quarantine and improved hygiene). To recognize and combat zoonotic diseases, the epidemiology of these infections must be understood. We need to identify pathogens, their vertebrate hosts, and their methods of transmission. Identifi cation should include knowledge of spatiotemporal disease patterns and their changes over time. These features can be used to identify dynamic processes involved in pathogen transmission, which 
can be used to account for observed disease patterns and ultimately forecast spread and establishment into new areas. Armed with information on expected disease patterns, we can address whether change has occurred beyond that which would normally be expected. However, this analysis may not be suitably responsive to control new and emerging zoonoses. Improved detection may be achieved through use of syndromic approaches rather than searching for specific pathogens.

After reviewing those information the PRECEDE-PROCEED MODEL (Green and Kreuter, 2005) below can be considered as a model that can be used for both research and planning dealing with zoonoses problems.

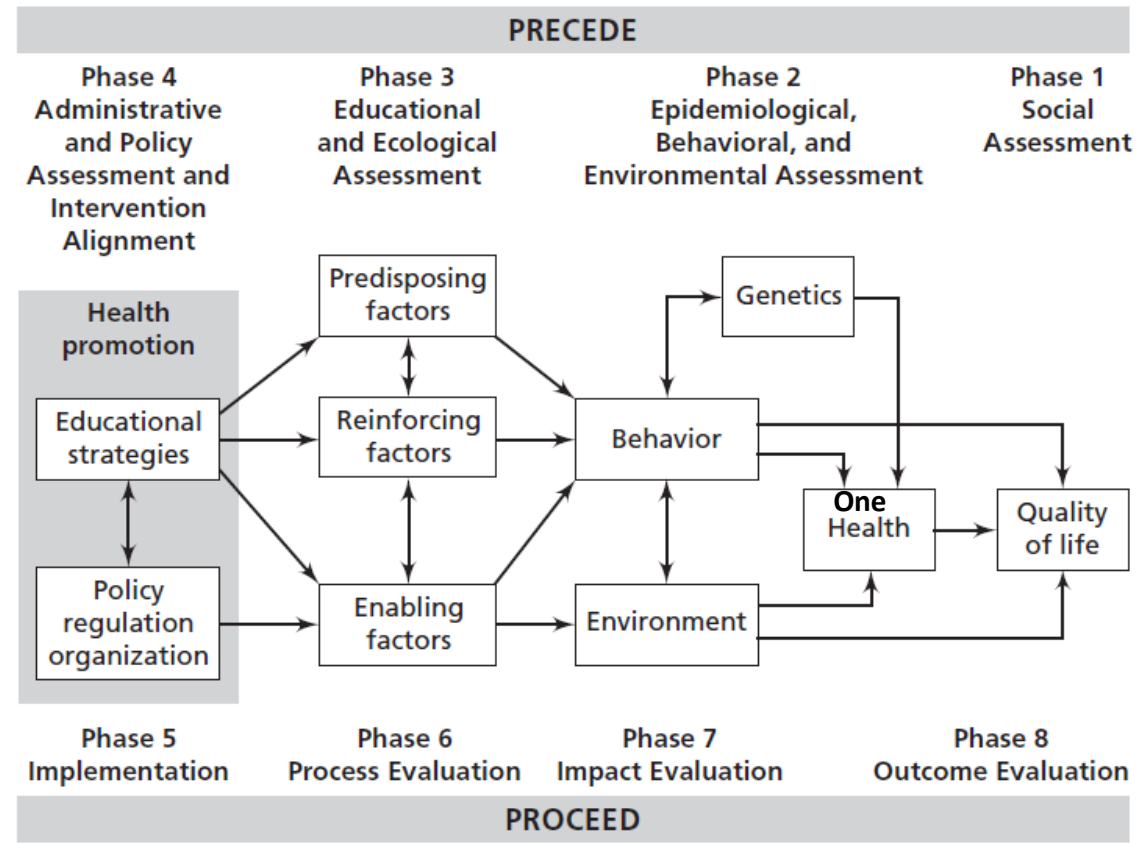

Figure 2. PRECEDE-PROCEED MODEL (modified) (Gielen, Mcdonald, Gary, Bone, 2008).

\section{Phase 1: Social Assessment, Participatory Planning, and Situation Analysis}

A social assessment is the "application, through broad participation, of multiple sources of information, both objective and subjective, designed to expand the mutual understanding of people regarding their aspirations for the common good" (Green and Kreuter, 2005). At this stage, the planners expand their understanding of the community in which they are working by conducting multiple data collection activities, such as interviews with key opinion leaders, focus groups with members of the community, observations, and surveys.

\section{Phase 2: Epidemiological, Behavioral, and Environmental Assessments}

This phase of the needs assessment identifies the health priorities and their behavioral and environmental determinants. Epidemiological Assessment. This analysis (1) identifies the health problems, issues, or aspirations on which the program will focus, (2) uncovers the behavioral and environmental factors most likely to influence the identified priority health issues, and (3) translates those priorities into measurable objectives for the program being developed (Green and Kreuter, 2005).

Behavioral Determinants. The behavioral determinants of a health problem can be understood on three levels. Most proximal are those behaviors or lifestyles that contribute to the occurrence and severity of a health problem (for example, eating undercooked chicken meat). The second, more distal determinant is the behavior of others who can directly affect the behavior of the individuals at risk (such as the mother does not know about zoonoses). The third and most distal behavioral determinant is the action of decision makers whose decisions affect the social or physical environment that influences the individuals at risk. 
Environmental Determinants. Environmental factors are those social and physical factors external to the individual, often beyond his or her personal control, that can be modified to support the behavior or influence the health outcome. Modifying environmental factors usually requires strategies other than education.

\section{Phase 3: Educational and Ecological Assessment}

After selecting the relevant behavioral and environmental factors for intervention, the framework directs planners to identify the antecedent and reinforcing factors that should be in place to initiate and sustain the change process. These factors are classified as predisposing, reinforcing, and enabling, and they collectively influence the likelihood that behavioral and environmental change will occur. "Predisposing factors are antecedents to behavior that provide the rationale or motivation for the behavior" (Green and Kreuter, 2005); they include individuals' knowledge, attitudes, beliefs, personal preferences, existing skills, and self-efficacy beliefs. "Reinforcing factors are those factors following a behavior that provide continuing reward or incentive for the persistence or repetition of the behavior" (Green and Kreuter, 2005). Examples include social support, peer influence, significant others, and vicarious reinforcement. "Enabling factors are antecedents to behavioral or environmental change that allow a motivation or environmental policy to be realized" (Green and Kreuter, 2005).

\section{Phase 4: Administrative and Policy Assessment and Intervention Alignment}

In Phase 4, the planner selects and aligns the program's components (that is, interventions) with the priority determinants of change previously identified. Its purpose is to identify resources, organizational barriers and facilitators, and policies that are needed for program implementation and sustainability.

\section{Phases 5-8: Implementation and Evaluation}

At this point, the health promotion program is ready for implementation (Phase 5). Data collection plans should be in place for evaluating the process, impact, and outcome of the program, which are the final three phases in the PRECEDE-PROCEED planning model (Phases 6-8). Typically, process evaluation determines the extent to which the program was implemented according to protocol. Impact evaluation assesses change in predisposing, reinforcing, and enabling factors, as well as in the behavioral and environmental factors. Finally, outcome evaluation determines the effect of the program on health and quality-of-life indicators.

\section{CONCLUSION}

Effective stakeholder engagement is key to the successful use of the One Health approach to address global health challenges. Identification of those stakeholders is the real challenge, as almost all sectors of civil society are and should be concerned about health and are, therefore, stakeholders in the development of effective solutions to the many problems that arise. Key to collaborative involvement is the development of trust and mutual respect across jurisdictional levels and disciplinary and political boundaries. Unfortunately, despite growing acknowledgement of the utility of the approach and the ample areas that could be improved through effective collaborations, there are numerous challenges threatening the implementation of One Health activities by stakeholders. These include a lack of consistent understanding of One Health and its potential benefits, sub-national priorities, disproportionate resource allocations, and conservatism. Other challenges to the implementation of One Health activities are: poor coordination amongst stakeholders, a lack of policy and legal frameworks for One Health, unwillingness to share data, inadequate human resources and laboratory capacity, the existence of many, and potentially diluting, One Health networks supported by external organisations, weak representation and engagement of stakeholders from the ecosystem/environment sector (Mazet, Uhart \& Keyyu, 2014). 


\section{REFERENCES}

Bidaisee, Satesh and Calum N. L. Macpherson. 2014. Zoonoses and One Health: A Review of the Literature Hindawi Publishing Corporation Journal of Parasitology Research Volume 2014, Article ID 874345, 8 pages http://dx.doi.org/10.1155/2014/874345

Cripps, Peter J., 2007. Acta Tropica 76 (2000) 77-80, Veterinary education, zoonoses and public health: a personal perspective

Cutler, Sally J., Anthony R. Fooks, and Wim H.M. van der Poel, 2010. Public Health Threat of New, Reemerging, and Neglected Zoonoses in the Industrialized World Emerging Infectious Diseases • www.cdc.gov/eid • Vol. 16, No. 1, January 2010

Galaz, V., Leach, M., Scoones, I. and Stein, C. (2015) The political economy of One Health research and policy, STEPS Working Paper 81 Brighton: STEPS Centre

Gielen, A.C.. E.M. Mcdonald, T. L. Gary, L. R. Bone, 2008. Using The Precede-Proceed Model To Apply Health Behavior Theories in Health Behavior And Health Education Theory, Research, And Practice, 4th Edition by Karen Glanz, Barbara K. Rimer, K. Viswanath, Editors, Copyright (C) 2008 By John Wiley \& Sons, Inc. All Rights Reserved.

Graham, J. P.. J. H. Leibler, L. B. Price et al., "The animal-human interface and infectious disease in industrial food animal production: rethinking biosecurity and biocontainment," Public Health Reports, vol. 123, no. 3, pp. 282-299, 2008.

Green, L. W., and Kreuter, M. W. Health Promotion Planning: An Educational and Ecological Approach. (4th ed.) New York: McGraw-Hill, 2005.

Mazet, J.A.K., M.M. Uhart \& J.D. Keyyu, 2014. Rev. sci. tech. Off. int. Epiz., 2014, 33 (2), $443-$ 452 Stakeholders in One Health.

National Institute Of Communicable Diseases, 2005. Zoonotic Diseases Of Public Health Importance Zoonosis Division National Institute Of Communicable Diseases (Directorate General Of Health Services) 22 - Sham Nath Marg, Delhi - 1100542005 Zoonosis Division National Institute Of Communicable Diseases (Directorate General Of Health Services) 22 Sham Nath Marg, Delhi - 1100542005

Pan American Health Organization, 2003. Zoonoses And Communicable Diseases Common To Man And Animals, Third Edition, Volume III: Parasitoses. Scientific And Technical Publication No. 580. Pan American Sanitary Bureau, Regional Office Of The World Health Organization 525 Twenty-Third Street, N.W. Washington, D.C. 20037 U.S.A.

Schneider MC, Aguilera XP, Smith RM, Moynihan MJ, Barbosa da Silva J Jr, Aldighieri S, et al., 2011. Importance of animal/human health interface in potential Public Health Emergencies of International Concern in the Americas. Rev Panam Salud Publica. 2011;29(5):371-9.

van der Giessen, J.W.B.. L.D. Isken, E.W. Tiemersma, 2004. Zoonoses in Europe a risk to public health. National Institute for Public Health and the Environment, Bilthoven, The Netherlands

World Health Organization, 2012. Research Priorities for Zoonoses and Marginalized Infections Technical report of the TDR Disease Reference Group on Zoonoses and Marginalized Infectious Diseases of Poverty (Technical report series ; no. 971)

WHO, Combating Emerging Infectious Diseases in the South East Asia Region, 2005, http://www.searo.who.int/entity/ emerging diseases/documents/SEA CD 139/en/index.html. 